\title{
Threshold characteristics and intensity fluctuations of lasers with excess quantum noise
}

\author{
M. A. van Eijkelenborg, ${ }^{*}$ M. P. van Exter, and J. P. Woerdman \\ Huygens Laboratory, Leiden University, P.O. Box 9504, 2300 RA Leiden, The Netherlands
}

(Received 31 July 1997; revised manuscript received 4 September 1997)

\begin{abstract}
We discuss the threshold characteristics and intensity noise of a laser with excess quantum noise as occurs, e.g., in an unstable-cavity laser. We give a theoretical description of the intensity aspects of excess noise based on laser rate equations, including bad-cavity effects. Experimentally, we have measured spectra of intensity noise and phase noise of small HeXe gas lasers. We operate the laser on either a stable or an unstable cavity, in order to change from a situation of no excess noise to large excess noise. By comparing the measured spectra with the theory, we deduce the excess-noise factor $K$ and the spontaneous-emission factor $\beta$. [S1050-2947(98)01401-2]

PACS number(s): 42.50.Lc, 42.60.Da, 42.55.Lt
\end{abstract}

\section{INTRODUCTION}

In lasers with nonorthogonal transverse eigenmodes the spontaneous-emission noise in the laser mode is enhanced by the transverse excess-noise factor, or $K$ factor [1-12]. Experimental values of transverse $K$ factors realized in unstable cavities range from $K=200$ to $500[2,6,7]$. The longitudinal $K$ factor, which arises due to nonorthogonality of the longitudinal eigenmodes, usually stays close to unity $[11,12]$. So far, all studies of the $K$ factor, both theoretically and experimentally, have concentrated on its consequences for the laser phase noise. In this paper we investigate, both theoretically and experimentally, the appearance of $K$ in the intensity noise. This automatically brings up the laser threshold characteristics, being intimately linked to intensity noise. In our analysis we include bad-cavity aspects since, in practical cases, excess noise occurs in lasers with relatively large losses, so that the cavity bandwidth often exceeds the gain bandwidth [13].

Setting up an appropriate quantum theory to describe excess quantum noise fluctuations is troubled by some conceptual difficulties. The excess noise arises in open-sided systems since the open character leads to nonorthogonal eigenmodes [1]. The standard descriptions of quantum noise in quantum optics rely on a complete set of orthogonal basis modes. For open systems there is no natural set of orthogonal modes [14]. The complex amplitudes of a set of nonorthogonal modes cannot be turned into a set of noncommuting operators because of problems related to unitarity and conservation of probability. Therefore, we have chosen to set up a phenomenological semiclassical model, based on rate equations for the laser intensity and population inversion, in which the consequences of mode nonorthogonality are inserted in an ad hoc fashion. We account for the excess spontaneous-emission noise by assigning to one of the photon emission channels, i.e., to the laser mode, a $K$ times higher weight than to the other modes. Our theoretical model is sufficiently general and simple that features of gas lasers, solid-state lasers (e.g., $\mathrm{Nd}: \mathrm{YVO}_{4}$ ), and semiconductor lasers (e.g., AlGaAs) can be easily incorporated. Experimentally,

*Electronic address: Eijkel@RuLhm1.LeidenUniv.nl we focus on the high-gain HeXe laser $(\lambda=3.51 \mu \mathrm{m})$, being a very suitable system for excess-noise measurements $[6,9,10]$. Using this laser, we directly compare the situation of no excess noise to large excess noise; this is done by changing the laser cavity from a stable to an unstable mirror configuration.

The paper is organized as follows. In Sec. II we present our theory. In Sec. III the experimental setup is described and in Sec. IV the experimental results. We end with a speculative discussion in Sec. V and a concluding summary in Sec. VI.

\section{THEORETICAL MODEL}

Our HeXe laser operates in the bad-cavity regime, in the sense that the decay rate of the cavity field is much larger than the collisional dephasing rate of the atomic polarization, i.e., the cavity bandwidth is much larger than the gain bandwidth. Therefore, the atomic polarization cannot be adiabatically eliminated $[13,15]$. In addition, the inversion cannot be adiabatically eliminated, as is evident from the pronounced relaxation oscillations of HeXe lasers. Generally, the combined dynamics of the electric field, the atomic polarization and the inversion is described by the Maxwell-Bloch equations $[16,17]$. In order to simplify this, the atomic polarization can be eliminated in a nonadiabatic way, where the badcavity effect is accounted for by Taylor expansion of the atomic susceptibility $\chi(\omega)$ around the laser frequency keeping only the first-order term and rewriting $d \chi / d \omega$ in the terms of the group refractive index $n_{\mathrm{gr}}[15,18]$. Incorporating $n_{\text {gr }}$ into the cavity loss rate changes the latter into the "dressed',-cavity loss rate. This procedure reduces the Maxwell-Bloch equations to a set of rate equations for the laser intensity and population inversion [19-21]

$$
\begin{gathered}
\dot{s}=\left[G(N)-\Gamma_{\mathrm{c}}(N)\right] s+R_{\mathrm{sp}}+f(t), \\
\dot{N}=\Lambda-\gamma_{0} N(1+\beta s),
\end{gathered}
$$

where $s$ is the number of photons in the lasing mode, $N$ is the inversion, i.e., the number of excited- minus ground-state atoms $\left(N=N_{2}-N_{1}\right), G(N)$ is the inversion-dependent intensity-gain rate, and $\Gamma_{\mathrm{c}}(N)$ is the cavity loss rate of the 
dressed cavity, which depends on the inversion $N$ through the group refractive index $n_{\mathrm{gr}}$. The dressed-cavity loss rate $\Gamma_{\mathrm{c}}(N)$ now contains the bad-cavity effects, taking into account the effect of the atomic polarization [18]. As an aside we note that for semiconductor lasers this complication is absent since there the gain bandwidth is much larger than the cavity bandwidth, so that the dressed-cavity loss rate $\Gamma_{\mathrm{c}}(N)$ equals the empty-cavity loss rate $\Gamma_{0}$. Spontaneous emission is included in the form of an average spontaneous-emission rate $R_{\mathrm{sp}}$ and a fluctuating term $f(t)$ [19-21]. The spontaneous-emission factor $\beta$ is defined as the fraction of spontaneous emission that ends up in the laser mode. $\Lambda$ is the pump rate (proportional to the injection current in case of semiconductor lasers or to the discharge power in case of gas lasers) and $\gamma_{0}$ is the decay rate of the inversion. Pump noise and spontaneous-emission noise in the inversion equation (1b) are neglected; this assumption is valid since for practical lasers $s \ll N$ so that the fluctuations in the photon number $s$ are dominant (i.e., we exclude the regime of a one-atom, one-photon laser).

By setting the stimulated emission rates in Eqs. (1a) and (1b) equal, we find the relation $G(N)=N \beta \gamma_{0}$. The decay rate of the inversion $\gamma_{0}$ can depend on the inversion $N$, as is the case for semiconductor lasers. Later on we will need the derivative of $G(N)$ with respect to $N$; therefore, we define the differential inversion decay rate $\gamma=\gamma_{0}+N\left(\partial \gamma_{0} / \partial N\right)$. For gas lasers and almost all solid-state lasers the decay is independent of the inversion, so that $\gamma=\gamma_{0}$.

The above rate equations (1a) and (1b) will now be modified ad hoc to our case of interest, i.e., we will include excess noise in a heuristic way. We assume that the atoms have $p=\beta^{-1}$ photon-emission channels available for spontaneous emission. It has been demonstrated recently that in case of phase noise, mode nonorthogonality increases the effect of spontaneous emission in the laser mode by the excess-noise factor, or $K$ factor $[2,3,6,9,10]$. We assume that the same holds in case of intensity noise, so that we simply account for possible mode nonorthogonality by giving one of the photon-emission channels, i.e., the lasing mode, a $K$ times higher weight than the others. Note that this weight factor only applies to the spontaneous-emission rate into the laser mode, but not to the stimulated emission rate. We stress that we treat the spontaneous-emission noise as appearing in Eq. (1a) in a perturbative sense, which in standard semiclassical laser theory requires $\beta \ll 1$; i.e., spontaneous emission in the laser mode is only a small fraction of the overall spontaneous emission. Since in our model $K \beta$ has taken the place of $\beta$ (see below), we have to assume $K \beta \ll 1$. This assumption is in fact reasonable for typical experiments reported so far, where, e.g., $K \approx 500, \beta \approx 4 \times 10^{-6}$ for a unstable-cavity semiconductor laser [7] or $K \approx 200, \beta \approx 1 \times 10^{-6}$ for a HeXe gas laser [6].

To account for possible population in the lower laser level $N_{1}$, we introduce, as usual, in the noise source $R_{\mathrm{sp}}$ the incomplete-inversion factor $N_{\mathrm{sp}}=N_{2} /\left(N_{2}-N_{1}\right)$ [13,22-25]. Using all this, we can write the average spontaneousemission rate into the lasing mode as $R_{\mathrm{sp}}=K \beta \gamma_{0} N_{2}$ or, more conveniently,

$$
R_{\mathrm{sp}}=K N_{\mathrm{sp}} \Gamma_{\mathrm{c}}
$$

where we have set $\Gamma_{\mathrm{c}}=G(N)$ and used the above derived relation $G(N)=N \beta \gamma_{0}$ and the definition of $N_{\mathrm{sp}}$. Note that above threshold the dressed-cavity loss rate $\Gamma_{\mathrm{c}}$ is independent of the pump rate due to gain clamping. The Langevin noise associated with this average spontaneous-emission rate is $\delta$ correlated in time and via the fluctuation-dissipation theorem found to be

$$
\begin{gathered}
\left\langle f\left(t^{\prime}\right) f\left(t^{\prime}+t\right)\right\rangle=2 R_{\mathrm{sp}} s \delta(t), \\
|\mathcal{F}(\omega)|^{2}=4 R_{\mathrm{sp}} s
\end{gathered}
$$

where $\mathcal{F}(\omega)$ is the Fourier transform of $f(t)$ [26]. The photon number $s$ occurs due to the admixture of the spontaneous-emission amplitude noise with the laser field as local oscillator [27].

We focus now on the appearance of the dressed-cavity loss rate $\Gamma_{\mathrm{c}}(N)$ in Eq. (1a). We consider a homogeneously broadened gain medium, with a Lorentzian gain spectrum with a full width at half maximum (FWHM) $\gamma_{\text {gain }} / \pi$. Note that the parameter $\gamma_{\text {gain }}$ can be quite different from $\gamma$ because the former is related to decay and dephasing, whereas the latter concerns only decay. For a bad-cavity laser above threshold, the dressed-cavity loss rate $\Gamma_{\mathrm{c}}$ has a natural upper limit $2 \gamma_{\text {gain }}$, the spectral width of the gain medium [13], whereas in the limiting case of no pumping, the dressedcavity loss rate must equal the empty-cavity loss rate $\Gamma_{0}$. Introducing the threshold inversion $N_{\text {th }}$, we find in fact for the dressed-cavity loss rate

$$
\Gamma_{\mathrm{c}}(N)=\frac{\Gamma_{0}}{n_{\mathrm{gr}}(N)}=\frac{\Gamma_{0}}{1+\left[\Gamma_{0} / 2 \gamma_{\mathrm{gain}}\right]\left[G(N) / G\left(N_{\mathrm{th}}\right)\right]} .
$$

This expression can be found in $[13,15]$, apart from the factor $G(N) / G\left(N_{\text {th }}\right)$, which has been introduced in order to include also the subthreshold behavior of $\Gamma_{\mathrm{c}}(N)$. This factor takes into account the dependence of the dispersion on the inversion through the Kramers-Kronig relation. Above threshold, the inversion is clamped, so that $G(N) / G\left(N_{\text {th }}\right)=1$; the factor $G(N) / G\left(N_{\text {th }}\right)$ is of importance only below threshold. If the gain is proportional to the inversion, $G(N) / G\left(N_{\text {th }}\right)$ can be written as $N / N_{\text {th }}$ and if, in addition, the inversion decay rate does not depend on the inversion, $G(N) / G\left(N_{\text {th }}\right)$ equals the dimensionless pump parameter $M=\Lambda / \Lambda_{\text {th }}$ [with the threshold pump rate $\Lambda_{\text {th }}=\gamma_{0}\left(N_{\text {th }}\right) N_{\text {th }}$ ] [28]. It can be easily checked that Eq. (4) has the proper limits; above threshold, when increasing $\Gamma_{0}$, the dressed-cavity loss rate has a natural upper limit $2 \gamma_{\text {gain }}$, whereas below threshold a decrease of the inversion leads to an increase of $\Gamma_{\mathrm{c}}(N)$ towards $\Gamma_{0}$. Note that for semiconductor lasers Eq. (4) is irrelevant since these lasers operate in the good-cavity regime $\left[\Gamma_{\mathrm{c}}(N)=\Gamma_{0}\right]$. Differentiating Eq. (4) with respect to $N$ we find, for operation close to threshold,

$$
\frac{\partial \Gamma_{\mathrm{c}}(N)}{\partial N}=-\frac{\Gamma_{\mathrm{c}}}{2 \gamma_{\text {gain }}} \frac{\partial G}{\partial N} \approx-\frac{1}{1+2 \gamma_{\text {gain }} / \Gamma_{0}} \frac{\partial G}{\partial N} .
$$

Far into the bad-cavity regime $\left(\Gamma_{0} \gg \gamma_{\text {gain }}\right)$ this results in $\partial \Gamma_{\mathrm{c}} / \partial N \approx-\partial G / \partial N$. For later use it is convenient to introduce a parameter $C$, which is defined as 


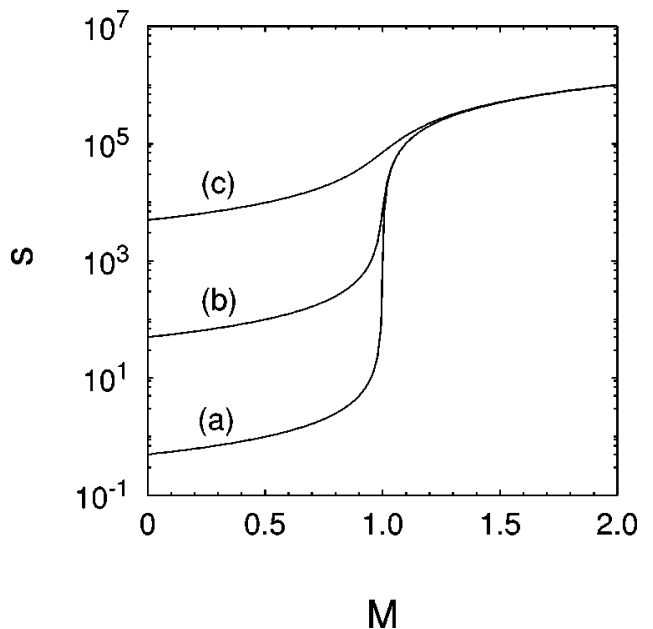

FIG. 1. Laser threshold characteristics in the presence of excess noise. The intracavity photon number $s$ is plotted versus the dimensionless pump parameter $M$. The drawn curves are calculated from Eq. (7) using $\beta=10^{-6}, C=2, N_{\mathrm{sp}}=1$, and $K=$ (a) 1 , (b) $10^{2}$, and (c) $10^{4}$. The presence of excess noise smoothens the input-output curve.

$$
C=\frac{2+2 \gamma_{\text {gain }} / \Gamma_{0}}{1+2 \gamma_{\text {gain }} / \Gamma_{0}}
$$

and obviously obeys $1<C<2$.

By setting the time derivatives in Eqs. (1a) and (1b) equal to zero, we can find the relation between the pump parameter $M$ and photon number $s$. We use $\Gamma_{\mathrm{c}}=\beta \Lambda_{\text {th }}$ and expand $G(N)-\Gamma_{\mathrm{c}}(N)$ around $N_{\mathrm{th}}$, with $G\left(N_{\mathrm{th}}\right)-\Gamma_{\mathrm{c}}\left(N_{\mathrm{th}}\right)=0$. Using Eq. (5) and $s \beta \ll 1$ we find

$$
s_{0}=\frac{1}{2 \beta}\left[(M-1)+\sqrt{(M-1)^{2}+4 K \beta \frac{N_{\mathrm{sp}}}{C}}\right] .
$$

Equation (7) shows the laser threshold behavior; the steadystate photon number is affected by the presence of excess noise in the sense that, as compared to the standard expression, $\beta$ is replaced by $K \beta$, as has been hinted at in [7]. This is not surprising, considering the fact that we have "by hand" multiplied $R_{\mathrm{sp}}$ with a factor of $K$ [cf. Eq. (2)]. Note the remarkable simplicity of Eq. (7); all complications due to the inversion dependence of gain and decay rates have disappeared. The photon number at threshold $s_{\text {th }}$ is given by

$$
s_{\mathrm{th}}^{2}=K \frac{N_{\mathrm{sp}}}{C \beta} .
$$

Notice that the excess-noise factor $K$ and the bad-cavity correction factor $C$ influence the threshold photon number $s_{\text {th }}$. A large excess-noise factor $K$ thus leads to an increase of $s_{\text {th }}$, increasing the laser output power at lasing threshold. To first order (i.e., $K \beta \ll 1$ ) the pump threshold pump rate $\Lambda_{\text {th }}$ will not be affected because this is dominantly determined by the spontaneous emission in the other (nonlasing) modes. Large excess-noise factors will smoothen the threshold transition. This can be seen in Fig. 1, where we have plotted $s_{0}$, as given by Eq. (7), versus $M$, using the values $\beta=10^{-6}, C=2, N_{\mathrm{sp}}=1$, and $K=$ (a) 1 , (b) $10^{2}$, and (c) $10^{4}$.
To calculate the intensity noise of the laser we will linearize Eqs. (1a) and (1b) around the operating point $N_{0}, s_{0}$. This linearization is reasonably safe far below threshold, because saturation is then relatively unimportant, and far above threshold, because the laser intensity is then relatively stable [21]. Linearization is of course bound to break down very close to the lasing threshold. We introduce the small fluctuations $\sigma$ and $\eta$, so that $s=s_{0}+\sigma$ and $N=N_{0}+\eta$, to obtain

$$
\begin{gathered}
\dot{\sigma}=-\frac{R_{\mathrm{sp}}}{s_{0}} \sigma+\gamma C \beta s_{0} \eta+f(t), \\
\dot{\eta}=-\Gamma_{\mathrm{c}} \sigma-\gamma\left(1+\beta s_{0}\right) \eta .
\end{gathered}
$$

We note that the differential inversion decay rate $\gamma$ enters these equations instead of the inversion decay rate $\gamma_{0}$.

Solution of Eqs. (9a) and (9b) by a Fourier transform is straightforward. We obtain the following power spectrum of the intensity noise:

$$
|\sigma(\omega)|^{2}=4 R_{\mathrm{sp}} s /\left|-i \omega+K \frac{N_{\mathrm{sp}} \Gamma_{\mathrm{c}}}{s_{0}}+\frac{\gamma C \beta s_{0} \Gamma_{\mathrm{c}}}{\gamma\left(1+\beta s_{0}\right)-i \omega}\right|^{2},
$$

which shows that the excess-noise factor $K$ and the spontaneous-emission factor $\beta$ are present in different terms of the denominator; this allows an independent measurement of these parameters. The interpretation of Eq. (10) is troubled due to the complicated nature of the denominator. However, the result simplifies considerably in the three limits that are discussed in Secs. II A-II C.

We note that in the comparison between theory and experiment one relies on the relation between intracavity photon number $s_{0}$ and laser output power $P_{\text {out }}$, which is

$$
P_{\text {out }}=h \nu \Gamma_{\mathrm{m}} s_{0},
$$

where we have introduced the dressed output-mirror transmission loss rate $\Gamma_{m}=n_{\mathrm{gr}}^{-1}(c / 2 L) \ln R$, with $R$ the outcoupling mirror reflectivity (the mirror loss rate is not necessarily equal to the cavity loss rate $\Gamma_{\mathrm{c}}, \Gamma_{\mathrm{m}} \leqslant \Gamma_{\mathrm{c}}$ ).

\section{A. Intensity noise at low frequency}

The low-frequency intensity noise is easily found by taking the limit $\omega \downarrow 0$ in Eq. (10) which gives

$$
|\sigma(0)|^{2}=\frac{4}{C \beta \Gamma_{\mathrm{c}}} s_{0} /\left(\frac{s_{\mathrm{th}}}{s_{0}}+\frac{s_{0}}{s_{\mathrm{th}}}\right)^{2}
$$

where $s_{\text {th }}$ is the photon number at threshold, as given by Eq. (8). The low-frequency intensity noise $|\sigma(0)|^{2}$ increases steeply as $s_{0}^{3}$ far below the lasing threshold, whereas it decreases as $s_{0}^{-1}$ far above threshold. Note that Eq. (12) does not depend on the damping rate $\gamma$. Experimentally, it might be difficult to find the precise position of the "kink" in the input-output characteristic that corresponds with threshold (see Fig. 1). Equation (12) provides a much easier way to find the laser threshold, namely, by determining at which output power the low-frequency noise strength $|\sigma(0)|^{2}$ is maximum. We will deduce the value of $s_{\text {th }}$ by fitting Eq. (12) 
to measurements of $|\sigma(0)|^{2}$ as a function of output power; this yields the value of $K \beta^{-1}$ [using Eq. (8) and the calculated values of $C$ and $\left.N_{\mathrm{sp}}\right]$.

\section{B. Intensity noise far below threshold}

Far below threshold, at small photon numbers $s_{0}$, the third term in the denominator of Eq. (10) can be neglected so that

$$
|\sigma(\omega)|^{2}=\frac{4 s_{0}^{2} \Delta \omega}{\omega^{2}+\Delta \omega^{2}} \quad \text { with } \Delta \omega=K \frac{N_{\mathrm{sp}} \Gamma_{\mathrm{c}}}{s_{0}} .
$$

This spectrum is Lorentzian with a half-width at half maximum $\Delta \omega$. Note that the bad-cavity correction factor $C$ plays no role in the subthreshold noise spectra. From Eq. (13) we see that, for the same number of photons in the lasing mode, a laser with $K \gg 1$ will have a much broader subthreshold intensity-noise spectrum than a laser with $K=1$. Experimentally, we will derive the value of $K$ from the width of the subthreshold Lorentzian noise spectra, combined with measurements of the dressed-cavity decay rate and the output power. By comparing the experimental determination of $K \beta^{-1}$ from the low-frequency intensity-noise measurements of Sec. II A to the value of $K$ determined with the subthreshold Lorentzian noise spectra, we will obtain a value for $\beta$.

We note that integration over the subthreshold spectrum in Eq. (13) gives

$$
\frac{1}{2 \pi} \int_{0}^{\infty}|\sigma(\omega)|^{2} d \omega=s_{0}^{2},
$$

which expresses that the mean square of the intensity fluctuations is as large as the square of the average intensity, as expected for "thermal'" light [29].

\section{Intensity noise far above threshold}

Far above threshold, at large photon numbers $s_{0}$, the second term in the denominator of Eq. (10), which scales as $s_{0}^{-1}$, can be neglected as compared to the other terms. We then find

$$
|\sigma(\omega)|^{2}=4 R_{\mathrm{sp}} s \frac{\gamma_{\mathrm{d}}^{2}+\omega^{2}}{\left(\omega^{2}-\omega_{0}^{2}\right)^{2}+\gamma_{\mathrm{d}}^{2} \omega^{2}}
$$

where we have introduced the relaxation-oscillation frequency $\omega_{0}$ by $\omega_{0}^{2}=\gamma C \beta s_{0} \Gamma_{\mathrm{c}}$ and the damping rate $\gamma_{\mathrm{d}}=\gamma\left(1+\beta s_{0}\right)$. Equation (15) has a limited validity for our case since in the HeXe laser severe complications of the relaxation-oscillation spectra may arise. As one example, a transversely nonuniform gain distribution, as is to be expected in a discharge tube, strongly alters the relaxationoscillation frequency [30]. As another example, in our present experiments we have observed a strong effect of nonlinear gain on the damping of the relaxation oscillations. We discuss now, as a small side step, the latter effect.

For many lasers the gain $G(N)$ is not only a function of the (saturated) inversion, but it also depends explicitly on the intensity; this is called nonlinear gain. More specifically, the gain in the saturated system is generally lower than in the unsaturated system with the same inversion due to a reduc- tion of the overlap between the mode and the gain medium, either in a spatial or in a spectral sense $[31,32]$. In a gas laser, spectral hole burning may occur for a Doppler-broadened gain transition and spatial hole burning may occur if the spatial diffusion of the atoms is sufficiently slow. In fact, by fitting Eq. (15) to the measured relaxation-oscillation spectra, we found that nonlinear gain is quite important in our gas lasers; it has a profound effect on the damping of the relaxation oscillations. For relatively small photon numbers the damping rate shows a strong increase with photon number $s_{0}$, which cannot be accounted for by $\gamma_{d}=\gamma\left(1+\beta s_{0}\right)$. Subsequently, for higher values of $s_{0}$ the damping rate saturates at a value that is more than an order of magnitude larger than the starting value $\gamma$ at $s_{0}=0$. This behavior is similar to that recently reported for a semiconductor laser [33]. We have found that these effects of nonlinear gain are larger for the stable- than for the unstable-cavity laser. This is to be expected since the stable-cavity laser has a smaller mode volume than the unstable-cavity laser, so that a certain photon number $s_{0}$ corresponds to a higher intracavity intensity. Finally, we note that all these complications concerning the relaxation oscillations do not affect Eqs. (12) and (13), which will play a key role in the analysis of our experimental data.

\section{EXPERIMENTAL SETUP}

Experimentally, we have not attempted to measure the input-output relation $s_{0}(M)$ as given by Eq. (7) and Fig. 1 since typically $\beta \approx 1 \times 10^{-6}$, so that the dimensionless width of the threshold transition is roughly $\sqrt{\beta} \approx 1 \times 10^{-3}$ [34], which demands an accuracy for $M$ better than $0.1 \%$. This cannot be realized experimentally due to fluctuations in the discharge power and due to the aging of the HeXe gas mixture (Xe depletion) [25]. Instead, we have focused on two methods, which are discussed below in Secs. IV A and IV B. We have compared measurements of intensity-noise spectra of a stable-cavity laser $(K=1)$ to those of an unstable-cavity laser $(K \gg 1)$. We use a small HeXe laser that operates in a single longitudinal and transverse mode (this applies to both the stable- and unstable-cavity regimes). A HeXe gas discharge is $\mathrm{rf}$ excited in a glass tube (5 $\mathrm{mm}$ inner diameter) providing an unsaturated gain of about $110 \mathrm{~dB} / \mathrm{m}$ at $\lambda=3.51$ $\mu \mathrm{m}$. The operating pressure is $0.5 \mathrm{kPa}$, which gives a FWHM gain bandwidth $\gamma_{\text {gain }} / \pi=152 \mathrm{MHz}$ [35] (including $110-\mathrm{MHz}$ Doppler broadening). This relatively narrow gain profile puts us well into the bad-cavity regime [in the experiments described below the measured group refractive index $\left(n_{\text {gr }}\right)$ is given by $n_{\text {gr }}=3.5$ for the stable and $n_{\mathrm{gr}}=6.8$ for the unstable cavity]. The inversion decay rate $\gamma=0.83 \times 10^{6} \mathrm{~s}^{-1}$, derived from the natural lifetime of $1.2 \mu \mathrm{s}$ found in literature [35]. The rf discharge is driven with an $L C$ circuit resonant at $15 \mathrm{MHz}$. The gain tube is terminated by two 0.5 -mm-thick quartz windows, each of which has a measured single-pass transmission of 0.91 .

As shown schematically in Fig. 2, the resonator has a length $L \approx 10 \mathrm{~cm}$ and consists of a concave dielectric output mirror $M_{1}$, with a $30-\mathrm{cm}$ radius of curvature and a reflectivity of $32 \%$, and a gold-coated mirror $M_{2}$. A key point is that the curvature of $M_{2}$ is different in the two experiments. We 


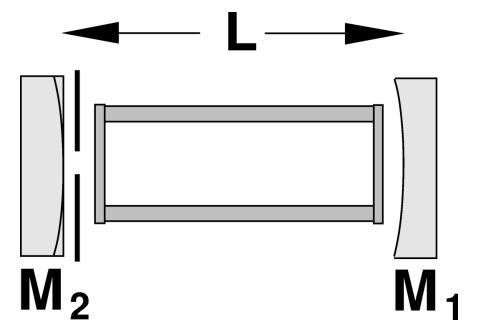

FIG. 2. Schematic drawing of the laser cavity. The two laser mirrors are labeled $M_{1}$ and $M_{2}$. The laser length is $L$. The screen just in front of mirror $M_{2}$ contains a square aperture with edge $2 a$.

take $M_{2}$ to be either a flat gold mirror, making the configuration a stable cavity, or a convex gold mirror with a $10.4-\mathrm{cm}$ radius of curvature, making it an unstable cavity. The linear round-trip magnification $M$ for the unstable case is 2.88 . As a limiting aperture we insert right in front of mirror $M_{2}$ a screen with a square aperture (as was used previously in $[6,9])$, with an area of $1.27 \times 1.28 \mathrm{~mm}^{2}$. This gives an equivalent Fresnel number $N=1.137$ [36]. Using the magnification $M$ and Fresnel number $N$ we calculated an excessnoise factor $K=82$ for the unstable-cavity case [37]. The stable-cavity laser has an (almost negligible) longitudinal excess-noise factor $K=1.1[11,12]$. The HeXe gain tube has a square shape with an inner area of $5 \times 5 \mathrm{~mm}^{2}$; this value is large enough to ensure that the laser mode remains clear of the glass tube at all times.

The laser output is split into two parts by a flat mirror with 90\% reflectivity (not drawn in Fig. 2). The transmitted part is measured by a room-temperature InAs detector, in order to determine the laser output power $P_{\text {out }}$. Using Eq. (11) this is converted into the intracavity photon number $s_{0}$. The reflected part is directed to a cryogenic InSb detector with a 4-MHz bandwidth, which is used for measuring the intensity-noise spectra. The relatively narrow gain bandwidth mentioned above ensures single longitudinal- and transversemode operation, since the transverse- and longitudinal-mode splittings are larger than the gain bandwidth, both for the stable and for the unstable cavity. In the case of the stablecavity laser, single transverse-mode operation is further established by the mode discrimination of the aperture.

For the stable-cavity laser the dressed-cavity loss rate can be calculated from the known mirror reflectivities and the transmission of the gain-tube windows. For the unstablecavity laser we measure the dressed-cavity loss rate by applying an axial magnetic field and determining the cavity

TABLE I. Summary of the various laser cavity parameters such as the radius of curvature of the mirrors $R_{1}$ and $R_{2}$ (the mirror radii are positive for convex curvature), the laser length $L$, the dressed cavity loss rate $\Gamma_{\mathrm{c}}$, the dressed mirror loss rate $\Gamma_{\mathrm{m}}$, the bad-cavity correction factor $C$, and the incomplete-inversion factor $N_{\mathrm{sp}}$.

\begin{tabular}{lccccccc}
\hline \hline $\begin{array}{l}\text { Laser } \\
\text { cavity }\end{array}$ & $\begin{array}{c}R_{1} \\
(\mathrm{~cm})\end{array}$ & $\begin{array}{c}R_{2} \\
(\mathrm{~cm})\end{array}$ & $\begin{array}{c}L \\
(\mathrm{~cm})\end{array}$ & $\begin{array}{c}\Gamma_{\mathrm{c}} \\
\left(10^{8} \mathrm{~s}^{-1}\right)\end{array}$ & $\begin{array}{c}\Gamma_{\mathrm{m}} \\
\left(10^{8} \mathrm{~s}^{-1}\right)\end{array}$ & $C$ & $N_{\mathrm{sp}}$ \\
\hline stable & -30 & $\infty$ & 9.49 & 6.84 & 5.11 & 1.72 & 1.4 \\
unstable & -30 & +10.4 & 9.40 & 8.14 & 2.67 & 1.85 & $1.4-2.2$ \\
\hline \hline
\end{tabular}
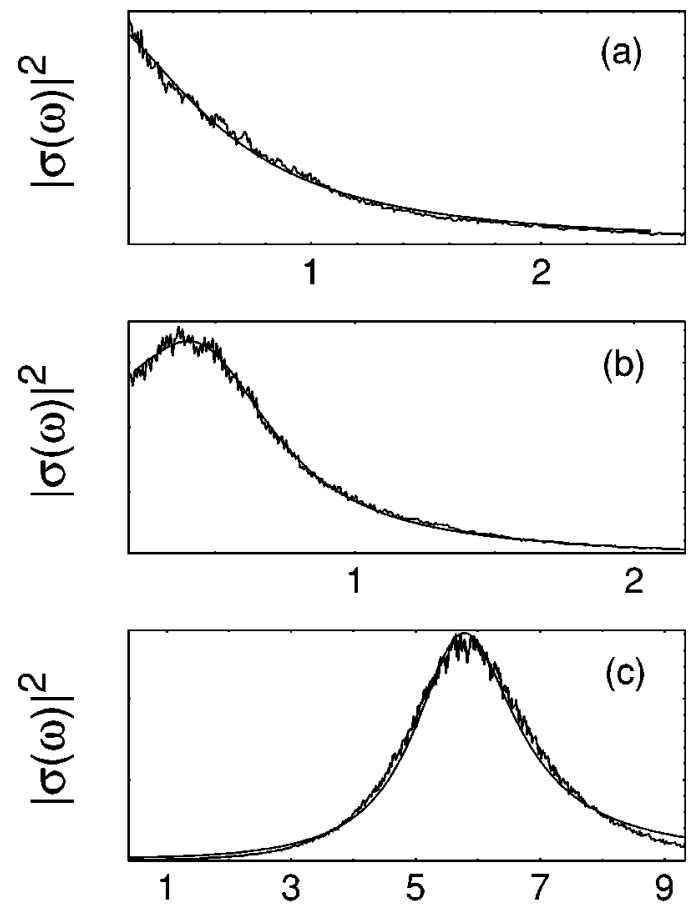

Frequency $\omega / 2 \pi \quad(\mathrm{MHz})$

FIG. 3. Typical intensity-noise spectra $|\sigma(\omega)|^{2}$, measured for the unstable-cavity laser (a) below threshold, $P_{\text {out }}=0.12 \mu \mathrm{W}$, (b) around threshold, $P_{\text {out }}=0.18 \mu \mathrm{W}$, and (c) above threshold, $P_{\text {out }}=10.3 \mu \mathrm{W}$. At threshold $P_{\text {out }}=0.24 \mu \mathrm{W}$ (as determined from Fig. 4).

mode-pulling strength $[38,39]$. The applied magnetic field induces a Zeeman splitting of the gain transition, which leads to oppositely directed mode pulling on the left and right circularly polarized $\left(\sigma_{+}\right.$and $\left.\sigma_{-}\right)$cavity modes. The strength of the frequency pulling depends on the cavity loss rate. The beat frequency of the $\sigma_{+}$and $\sigma_{-}$modes is recorded by a detector behind a linear polarizer; this frequency reflects the mode-pulling strength and thus provides a value for the dressed-cavity loss rate.

Finally, we will discuss the behavior of the incompleteinversion factor $N_{\text {sp }}$. It has been shown by Kuppens et al. [25] that for small HeXe lasers as we use, the incompleteinversion factor $N_{\mathrm{sp}}$ increases almost linearly with the discharge power $P_{\mathrm{rf}}$, as $N_{\mathrm{sp}}=1.2+0.26 P_{\mathrm{rf}}$, with $P_{\mathrm{rf}}$ expressed in watts. For the subthreshold measurements, we use a small discharge power $P_{\mathrm{rf}} \leqslant 1 \mathrm{~W}$; this gives $N_{\mathrm{sp}} \approx 1.4$ for both the stable- and unstable-cavity cases. This value of $N_{\mathrm{sp}}$ will be used in Secs. IV A and IV B for both the stable- and unstable-cavity lasers. For the above-threshold phase-noise measurements on the unstable-cavity laser, a somewhat larger discharge power $\left(P_{\mathrm{rf}} \approx 5 \mathrm{~W}\right)$ was needed to bring the laser above threshold, so that the incomplete-inversion factor is somewhat larger, $N_{\mathrm{sp}} \approx 2.2$. This value is used for the unstable-cavity laser measurements in Sec. IV C. For the stable-cavity measurements in Sec. IV C we use again the above value $N_{\mathrm{sp}} \approx 1.4$ since $P_{\mathrm{rf}} \leqslant 1 \mathrm{~W}$.

The above-mentioned experimental details, the measured dressed-cavity loss rates $\Gamma_{\mathrm{c}}$, the bad-cavity correction fac- 


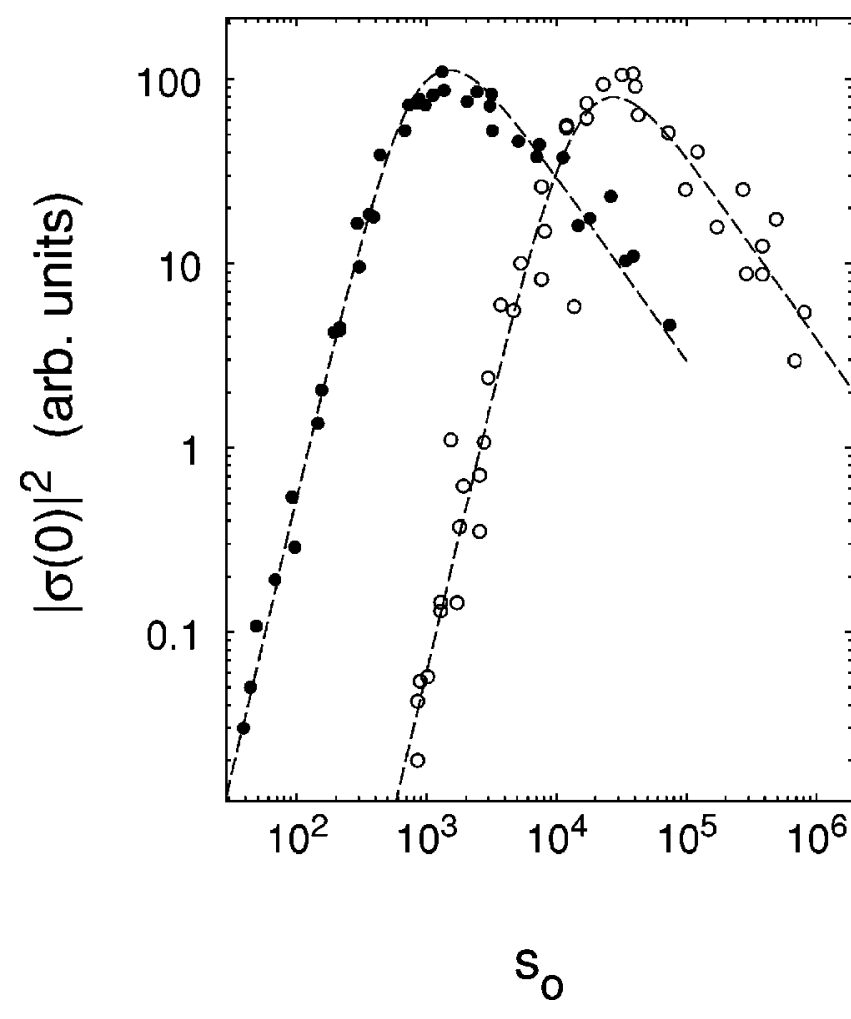

FIG. 4. Intensity-noise strength at low frequency $|\sigma(0)|^{2}$ versus the number of photons $s_{0}$ in the laser cavity. We show results for both the stable cavity (filled circles) and unstable cavity (open circles). Both of the dashed curves are fits to Eq. (12), which yield $s_{\text {th }}$ for each case. We find $s_{\text {th }}=858 \pm 60$ for the stable-cavity laser (filled circles) and $s_{\mathrm{th}}=(15.9 \pm 1.5) \times 10^{3}$ for the unstable-cavity laser (open circles). This corresponds to output powers $P_{\mathrm{th}}=0.025 \pm 0.002 \mu \mathrm{W}$ and $P_{\mathrm{th}}=0.24 \pm 0.02 \mu \mathrm{W}$, respectively.

tors $C$, and the incomplete-inversion factor $N_{\mathrm{sp}}$ are summarized in Table I.

\section{EXPERIMENTAL RESULTS}

Typical examples of measured intensity-noise spectra are shown in Fig. 3 for the case of the unstable-cavity laser. All measured spectra have been corrected for the 4-MHz bandwidth of the detector. In Fig. 3(a) we show an intensity-noise spectrum measured below threshold; the drawn curve is a fit to Eq. (13). Figure 3(c) shows a spectrum measured far above threshold. The drawn curve is a fit to Eq. (15). Very close to threshold we measure spectra such as shown in Fig. 3(b), using Eq. (15) as the fit curve. It is clear from Fig. 3 that the behavior of the spectra when going through threshold nicely follows the calculations. We will now proceed with methods proposed in Secs. II A and II B to analyze the experimental results, i.e., we will measure both the lowfrequency noise and the width of the subthreshold Lorentzian spectra as a function of $P_{\text {out }}$.

\section{A. Analysis of intensity noise at low frequency}

From the measured spectra we determine the lowfrequency noise level $|\sigma(0)|^{2}$ by taking the value at $\omega / 2 \pi=280 \mathrm{kHz}$ (to avoid the low-frequency technical-noise peak around zero frequency). The measured output power

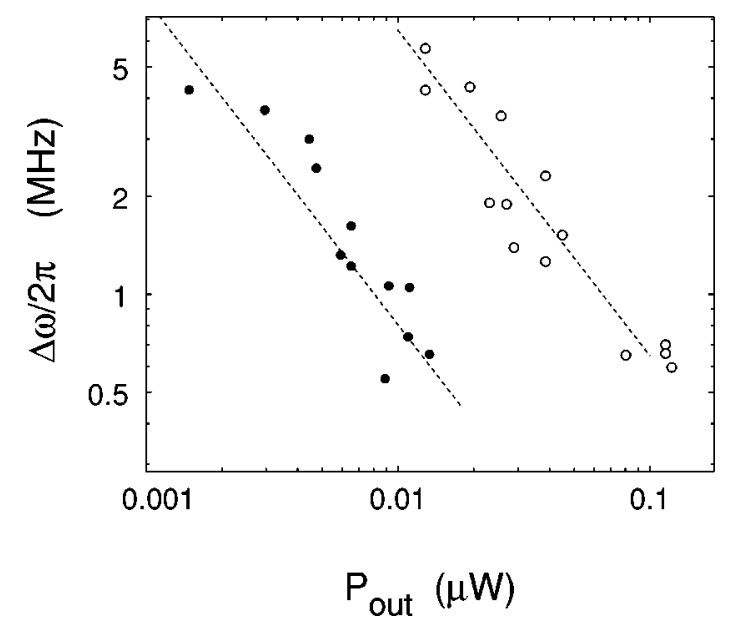

FIG. 5. Measured Lorentzian widths $\Delta \omega / 2 \pi$ of the subthreshold intensity-noise spectra as a function of the laser output power $P_{\text {out }}$ for both the stable (filled circles) and the unstable cavity (open circles). The dotted fitting curves represent Eq. (13), with $s_{0}$ converted into $P_{\text {out }}$ using Eq. (11).

$P_{\text {out }}$ is converted into an intracavity photon number using Eq. (11). The resulting curve of $|\sigma(0)|^{2}$ versus $s_{0}$ is shown in Fig. 4 for both the stable- (filled circles) and the unstablecavity case (open circles). The dashed curves are fits to Eq. (12), which nicely follow the data points; below threshold the low-frequency noise level rises proportionally to $s_{0}^{3}$, whereas above threshold it reduces proportionally to $s_{0}^{-1}$. From both curves it can be estimated that we operate the laser rather close to threshold; the range of measurements corresponds to the photon number $s_{0}$ varying from roughly a factor of 10 below to a factor of 10 above the threshold photon number. Expressed in $M$ this corresponds to the range $M=0.99-1.01$ (assuming $\beta \sim 10^{-6}$ ). This close proximity to threshold ensures that we detect the noise of a single laser mode only, the higher-order modes being much further below threshold.

The fitting of Eq. (12) to the data in Fig. 4 provides the value of $s_{\text {th }}$ for both cases. We find $s_{\text {th }}=858 \pm 60$ for the stable-cavity laser and $s_{\text {th }}=(15.9 \pm 1.5) \times 10^{3}$ for the unstable-cavity laser. This difference can be ascribed to the difference of the excess-noise factor $K$ for the two lasers and the difference in $\beta$. Using Eq. (8) and the values in Table I we find $K \beta^{-1}=(9.3 \pm 1.4) \times 10^{5}$ for the stable and $K \beta^{-1}=(3.4 \pm 0.7) \times 10^{8}$ for the unstable cavity. In Sec. IV $B$ we will compare these values with independent measurements of $K$ to obtain a value for $\beta$.

\section{B. Analysis of intensity noise far below threshold}

The subthreshold Lorentzian spectrum of Eq. (13) has been fitted to data as shown in Fig. 3(a). The fitting results for the width $\Delta \omega / 2 \pi$ are shown in Fig. 5 as a function of $P_{\text {out }}$ for the stable-cavity (filled circles) and the unstablecavity laser (open circles). For the stable cavity we find $(\Delta \omega / 2 \pi) P_{\text {out }}=(8.0 \pm 1.0) \times 10^{-3} \mathrm{~Hz} \mathrm{~W}$ and for the unstable cavity $(\Delta \omega / 2 \pi) P_{\text {out }}=(65 \pm 9) \times 10^{-3} \mathrm{HzW}$. Using Eq. (13), Eq. (11), and the values of the dressed-cavity loss rate $\Gamma_{\mathrm{c}}$ in Table I, we find $K=1.9 \pm 0.3$ for the stable cavity and $K=24 \pm 4$ for the unstable cavity. As expected, the unstable- 
TABLE II. Summary of the various experimentally determined values. The bottom row indicates in which section the results were obtained.

\begin{tabular}{lcccccc}
\hline \hline $\begin{array}{l}\text { Laser } \\
\text { cavity }\end{array}$ & $\begin{array}{c}K \\
\text { Calculation }\end{array}$ & $\begin{array}{c}K \\
\text { Subthreshold }\end{array}$ & $\begin{array}{c}K \beta^{-1} \\
\text { Low frequency }\end{array}$ & $\begin{array}{c}K \\
\text { Phase noise }\end{array}$ & $\begin{array}{c}\beta \\
\text { Combining } K \text { and } K \beta^{-1}\end{array}$ & $\begin{array}{c}\beta \\
\text { Calculation }\end{array}$ \\
\hline Stable & 1.1 & $1.9 \pm 0.3$ & $(9.3 \pm 1.4) \times 10^{5}$ & $1.1 \pm 0.2$ & $2.0 \times 10^{-6}$ & $3.7 \times 10^{-6}$ \\
Unstable & 82 & $24 \pm 4$ & $(3.4 \pm 0.7) \times 10^{8}$ & $32 \pm 5$ & $0.71 \times 10^{-7}$ & $(1.2-5.9) \times 10^{-7}$ \\
See Sec. & IV B & IV B & IV A & IV C & IV D & IV D \\
\hline \hline
\end{tabular}

cavity laser has a much larger excess-noise factor. These values can be compared with theoretical values: The stablecavity laser has a calculated longitudinal $K$ factor $K=1.1$ and the unstable-cavity laser a calculated transverse $K$ factor $K=82$. The agreement between the experiments and the calculated values of $K$ is no better than a factor of 3 . Note that deviations of this magnitude are commonly found when comparing excess-noise measurements to calculations $[2,3,6]$.

\section{Phase-linewidth measurements}

Values of $K$ can of course also be obtained from phaselinewidth measurements, as has been demonstrated before $[2,6,9,10]$. We determine the quantum-limited phase linewidth of our laser from the spectral width of the beat frequency between the $\sigma_{+}$and $\sigma_{-}$modes $[38,39]$, which was also used to measure the cavity loss rate (see Sec. III). In short, this linewidth measurement technique is based on the following idea. The combination of frequency-split $\sigma_{+}$and $\sigma_{-}$polarized light is equivalent to linearly polarized light with a rotating angle of polarization. This rotation is disturbed only by the randomly polarized spontaneous-emission noise since technical noise (such as mirror vibrations) has no effect on the laser polarization. By measuring the noise in the polarization-rotation frequency, we can directly obtain the quantum-limited laser linewidth. This has been described in detail in [39]. From the measured linewidth we deduce the excess-noise factor by comparing to the calculated linewidth of a stable-cavity laser with the same loss; the latter linewidth is well understood [13].

These measurements are similar to those reported in $[6,9,10,39,40]$, so we will be brief here. The measurements of the phase linewidth as a function of $P_{\text {out }}$ always showed the expected Schawlow-Townes $P_{\text {out }}^{-1}$ dependence, as observed before. The experimental result for $K$ obtained from the stable-cavity laser phase linewidth is $K=1.1 \pm 0.2$, in good agreement with the calculations mentioned in Sec. IV $\mathrm{B}$. The experimental result for the unstable cavity laser is $K=32 \pm 5$. We conclude, as in Sec. IV B, that the measured unstable-cavity value of $K$ is smaller than calculated, again by a factor of about 3 .

We summarize the various experimental results in Table II.

\section{Determination of the spontaneous-emission factor}

The independent determination of $K \beta^{-1}$ and $K$ from the intensity-noise measurements in Secs. IV A and IV B allows for a determination of the spontaneous-emission factor $\beta$. We divide the number in the second column of Table II, i.e., the measured value of $K$, by the number in the third column, i.e., the measured value of $K \beta^{-1}$, in order to obtain $\beta$. We find $\beta_{\text {stable }}=2.0 \times 10^{-6}$ for the stable-cavity laser and $\beta_{\text {unstable }}=7.1 \times 10^{-8}$ for the unstable-cavity laser. Clearly the unstable cavity has a much smaller spontaneous-emission factor $\beta$. According to theory, $\beta$ should vary inversely proportional to the mode volume [41]; i.e., one expects $\beta_{\text {unstable }}$ to be much smaller than $\beta_{\text {stable }}$, as measured, since the unstable-resonator laser operates with a much larger mode volume.

We theoretically estimate $\beta$ using Eq. (11) in [41], which requires that the effective mode volume $V_{\text {cav }}^{\text {eff }}$ is known. For the stable cavity this can be calculated; we find $V_{\text {cav }}^{\text {eff }}=\pi w_{0}^{2} L=4.7 \times 10^{-8} \mathrm{~m}^{3}$, so that $\beta=3.7 \times 10^{-6}$. This is a factor of 2 larger than the measured value. For the unstable cavity the effective mode volume is not properly defined. We estimate that $V_{\text {cav }}^{\text {eff }}$ is larger than $V_{\text {cav }}^{\text {eff }}=(2 a)^{2} L=1.5 \times 10^{-7}$ $\mathrm{m}^{3}$, where $2 a$ is the edge of the square aperture in Fig. 2, and smaller than $V_{\text {cav }}^{\text {eff }}=7.4 \times 10^{-7} \mathrm{~m}^{3}$, where the latter value is based on the entire volume that is covered by the rays of the geometrical eigenmode. These values of $V_{\text {cav }}^{\text {eff }}$ lead to $1.2 \times 10^{-7}<\beta<5.9 \times 10^{-7}$, which is somewhat larger than the experimental value $\beta_{\text {unstable }}=7.1 \times 10^{-8}$ found above.

\section{THRESHOLDLESS LASER?}

As we have stressed, our model is a phenomenological model to investigate the influence of excess noise on the threshold characteristics of a laser. One may of course question the validity of this model. However, we remind the reader that, as was discussed in the Introduction, a proper quantum theory is not available. This leaves some freedom for speculations that we will explore below.

Recently, the $\beta$ factor, i.e., the fraction of spontaneous emission radiated into a specific mode, has become of great importance in relation to the $\beta=1$ laser, sometimes called a zero-threshold laser $[42,43]$. A better terminology is thresholdless laser [34]. The current interpretation of the excessnoise factor $K$ implies that the fraction of spontaneous emission that ends up in the laser mode is enhanced by this factor, effectively enhancing $\beta$ by a factor of $K$. As mentioned in Sec. II, this interpretation is supported by experiments on phase noise of unstable-cavity lasers $[2,3,6,9,10]$. On the basis of our phenomenological model we find that the threshold characteristics of a large- $K$ laser have an appearance that approaches that of a thresholdless laser; the kink in the input output curve (see Fig. 1) will become smoother and smoother the larger $K$ becomes. Extrapolation of this scenario would provide an alternative route to reach threshold- 
less laser operation. The important parameter is now $K \beta$ instead of $\beta$.

The value of $\beta$ has the natural upper limit of 1 , which corresponds to all the spontaneous emission being directed into the laser mode. The limiting situation $\beta=1$ is notoriously difficult to realize since it requires a very small laser cavity volume. Therefore, it is tempting to use a large- $K$ factor for "leverage," maximizing $K \beta$ instead of $\beta$. The largest experimentally realized $K$ factor is $\sim 700$ [44]. Theoretically, there seems to be no limit to the value of $K$, and values as large as $10^{4}$ have been calculated for a onedimensional hard-edged unstable cavity laser [4], implying a value of $10^{8}$ for the corresponding two-dimensional cavity when using a square aperture [9]. Therefore, the product $K \beta$ seems unlimited. It remains to be seen, however, how realistic such gigantic $K$ factors are.

It should be noted that for $K \beta \gg 1$ the $K$ noise photons in the mode are in principle able to saturate the inversion since $p=\beta^{-1}$ is the saturation photon number. Therefore, it would be interesting to see what the photon statistics and phase coherence of a $K \beta \gg 1$ laser are.

Naively speaking, the case $K \beta \gg 1$ suggests an inconsistency: More than $100 \%$ of the spontaneous emission would end up in the laser mode. This, however, is not the case. A large- $K$ factor arises when the laser eigenmodes are highly nonorthogonal, so that a substantial degree of overlap between different transverse modes can be found [1]. The noise in different modes is then strongly correlated, so that after selection of one mode, i.e., the laser mode, there appears to be a factor of $K$ more spontaneously-emitted photons. However, the overall spontaneous-emission rate into all modes, including the laser mode, is unchanged [8]. When determining the fraction of the spontaneous emssion that ends up in the laser mode, this strong overlap betweeen all modes should be taken into account, removing the inconsistency.

We stress again that the case $K \beta \gg 1$, where these intriguing phenomena potentially occur, is beyond the validity range of our model (we have assumed $K \beta \ll 1$ ). Proper treat- ment of this case requires a fully quantum-mechanical theory.

\section{SUMMARY}

We have investigated, both theoretically and experimentally, the influence of excess noise on both the threshold characteristics and the intensity noise of a laser. Theoretically, we have presented laser rate equations for the photon number and atomic inversion, including an ad hoc excessnoise factor $K\left(K \ll \beta^{-1}\right)$. Also, we have included bad-cavity aspects such as the dependence of the cavity loss rate on inversion. We have found that, due to the presence of excess noise, the output power at lasing threshold is increased by a factor $\sqrt{K}$ and we have derived expressions for the subthreshold intensity-noise spectra, containing the excess-noise factor $K$. To first-order approximation $(K \beta \ll 1)$ there is no change in the laser threshold pumping condition.

Experimentally, we have studied intensity-noise spectra of small $\mathrm{HeXe}$ gas lasers, which operated on either a stable cavity (no excess noise) or an unstable cavity (large excess noise). From the measured low-frequency intensity-noise strength we deduced the value of $K \beta^{-1}$. Further, for a laser operating relatively far below threshold we observed that the intensity-noise spectrum is a Lorentzian, centered at zero frequency. The width of this spectrum was used to determine $K$. By combining these independently measured values of $K \beta^{-1}$ and $K$, we obtained a value for $\beta$. In most cases the agreement between experiment and theory was no better than a factor of 2 or 3; this is, however, typical for this kind of work $[2,6,9,10]$ and may be not surprising in view of the complexity of a real-life gas laser.

We have speculated on the possibility to reach thresholdless laser operation by maximizing the value of $K \beta$ and we indicated the need for a proper quantum theory. Developing such theory is highly nontrivial in view of the fact that we deal with a three-dimensional open-sided nonlinear system. As a first step in this direction, an interesting quantummechanical "toy model" has been reported very recently [45].
[1] A. E. Siegman, Phys. Rev. A 39, 1253 (1989); 39, 1264 (1989).

[2] Y. J. Cheng, C. G. Fanning, and A. E. Siegman, Phys. Rev. Lett. 77, 627 (1996).

[3] Y. J. Cheng, P. L. Mussche, and A. E. Siegman, IEEE J. Quantum Electron 30, 1498 (1994).

[4] G. H. C. New, J. Mod. Opt. 42, 799 (1995).

[5] M. A. Rippin and G. H. C. New, J. Mod. Opt. 43, 993 (1996).

[6] M. A. van Eijkelenborg, Å. M. Lindberg, M. S. Thijssen, and J. P. Woerdman, Phys. Rev. Lett. 77, 4314 (1996).

[7] G. Yao, Y. C. Chen, C. M. Harding, S. M. Sherrick, R. J. Dalby, R. G. Waters, and C. Largent, Opt. Lett. 17, 1207 (1992).

[8] H. A. Haus and S. Kawakami, IEEE J. Quantum Electron. 21, 63 (1985).

[9] M. A. van Eijkelenborg, Å. M. Lindberg, M. S. Thijssen, and J. P. Woerdman, Opt. Commun. 137, 303 (1997).

[10] M. A. van Eijkelenborg, Å. M. Lindberg, M. S. Thijssen, and
J. P. Woerdman, Phys. Rev. A 55, 4556 (1997).

[11] W. A. Hamel and J. P. Woerdman, Phys. Rev. Lett. 13, 1506 (1990).

[12] W. A. Hamel and J. P. Woerdman, Phys. Rev. A 40, 2785 (1989).

[13] M. P. van Exter, S. J. M. Kuppens, and J. P. Woerdman, Phys. Rev. A 51, 809 (1995).

[14] I. H. Deutsch, J. C. Garrison, and E. M. Wright, J. Opt. Soc. Am. B 8, 1244 (1991).

[15] S. J. M. Kuppens, M. P. van Exter, and J. P. Woerdman, Phys. Rev. Lett. 72, 3815 (1994).

[16] M. I. Kolobov, L. Davidovich, E. Giacobino, and C. Fabre, Phys. Rev. A 47, 1431 (1993).

[17] L. A. Lugiato, P. Mandel, and L. M. Narducci, Phys. Rev. A 29, 1438 (1984).

[18] The frequency dependence of $\chi$ implies in the time domain that the induced atomic polarization is not simply proportional to the electric field; there is a memory effect [15]. 
[19] C. H. Henry, IEEE J. Quantum Electron. 19, 1391 (1983).

[20] M. P. van Exter, W. A. Hamel, J. P. Woerdman, and B. R. P. Zeijlmans, IEEE J. Quantum Electron. 28, 1470 (1992).

[21] D. E. McCumber, Phys. Rev. 141, 306 (1966).

[22] P. Goldberg, P.W. Milonni, and B. Sundaram, Phys. Rev. A 44, 1969 (1991).

[23] M. Lax, in Physics of Quantum Electronics, edited by P. L. Kelley, B. Lax, and P. E. Tannenwald (McGraw-Hill, New York, 1966).

[24] H. Haken, Z. Phys. 190, 327 (1966).

[25] S. J. M. Kuppens, M. A. van Eijkelenborg, C. A. Schrama, M. P. van Exter, and J. P. Woerdman, IEEE J. Quantum Electron. 32, 383 (1996).

[26] In going from Eq. (3a) to Eq. (3b) we acquire a factor of 2 since by $\omega$ we mean $|\omega|$, instead of having positive- and negative-frequency components.

[27] C. H. Henry, IEEE J. Quantum Electron. 18, 259 (1982).

[28] In a cavity with large localized losses, i.e., with an appreciable longitudinal Petermann factor [11,12], Eq. (4) is slightly more complicated due to the nonuniformity of the longitudinal intensity distribution [see Eq. (21) of [13]].

[29] R. Loudon, The Quantum Theory of Light (Oxford University Press, New York, 1991).

[30] Y. J. Cheng, P. L. Mussche, and A. E. Siegman, IEEE J. Quantum Electron. 31, 391 (1995).

[31] M. Asada and Y. Suematsu, IEEE J. Quantum Electron. 21, 434 (1985).
[32] M. Willatzen, A. Uskov, J. Mørk, H. Olesen, B. Tromborg, and A. P. Jauho, IEEE Photonics Technol. Lett. 3, 606 (1991).

[33] H. Li, IEEE Photonics Technol. Lett. 8, 1594 (1996).

[34] P. R. Rice and H. J. Carmichael, Phys. Rev. A 50, 4318 (1994).

[35] R. Vetter and E. Marié, J. Phys. B 11, 2845 (1978).

[36] A. E. Siegman, Lasers (University Science Books, Mill Valley, CA, 1986).

[37] We calculated the $K$ factor with the commercially available software vSOURCE, written by J. L. Doumont, available from Sciopt Enterprises, San Jose, CA.

[38] M. A. van Eijkelenborg, C. A. Schrama, and J. P. Woerdman, Opt. Commun. 124, 462 (1996).

[39] A. M. Lindberg, M. A. van Eijkelenborg, and J. P. Woerdman, IEEE J. Quantum Electron. 33, 1767 (1997).

[40] M. A. van Eijkelenborg, C. A. Schrama, and J. P. Woerdman, Opt. Commun. 119, 97 (1995).

[41] M. P. van Exter, G. Nienhuis, and J. P. Woerdman, Phys. Rev. A 54, 3553 (1996).

[42] W. Mohideen, W. S. Hobson, S. J. Pearton, F. Ren, and R. E. Slusher, Appl. Phys. Lett. 64, 1911 (1994).

[43] J. P. Zhang, D. Y. Chu, S. L. Wu, S. T. Ho, W. G. Bi, C. W. Tu, and R. C. Tiberio, Phys. Rev. Lett. 75, 2678 (1995).

[44] M. A. van Eijkelenborg, Ph.D. thesis, University of Leiden, 1997 (unpublished).

[45] P. Grangier and J. P. Poizat, Eur. Phys. Rev. D (to be published). 